\title{
Unequal Error Protected Bitplanes Improve the Robustness of Wireless Holographic Image Communications
}

\author{
Yongkai Huo, ${ }^{a, b}$ Thomas J. Naughton, ${ }^{c}$ and Lajos Hanzo ${ }^{b}$ \\ ${ }^{a}$ College of Computer Science \& Software Engineering, Shenzhen University, China.

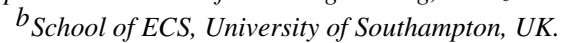 \\ ${ }^{c}$ Department of Computer Science, Maynooth University-National University of Ireland Maynooth, Ireland.

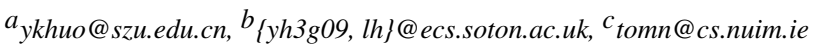

\begin{abstract}
Digital holograms are partitioned into multiple bitplanes that are independently encoded by a forward error correction code for transmission over wireless channels. PSNR improvements of $12.5 \mathrm{~dB}$ are achieved with a recursive systematic convolutional code.
\end{abstract}

OCIS codes: $090.1995,100.2000$

\section{Introduction}

Holography [1] constitutes a sophisticated technique of recording and reconstructing both the amplitude and phase of an optical wavefront relying on the interference and diffraction imposed by an object on visible light. Optical holography allows the holographic images to be recorded and reconstructed using a white-light illumination source [2] or a illuminating laser [3]. Digital holography (DH) [4,5] refers to the class of techniques that records an optical hologram digitally and reconstruct the image using numerical manipulations.

Digital holograms may be widely utilized in future applications. However, apart from [6], the transmission of digital holograms has rarely been researched. We propose an optimized unequal error protection based forward error correction (Opt-UEP-FEC) coded system, where the holograms will be transmitted bitplane by bitplane after forward error correction (FEC).

\section{System Architecture}

We introduce the proposed unequal error protection (UEP) based FEC coded (Opt-UEP-FEC) system conceived for holographic communications, whose system model is detailed in Fig. 1.

At the transmitter, the original hologram $U$ (for example, as shown in Fig. 2) is de-multiplexed into the classic bitplanes $u_{0}, \cdots, u_{m-1}$ by the $D E M U X$ block, where $u_{0} / u_{m-1}$ represents the most/least significant bitplane. Meanwhile, the original hologram $U$ is input to the "Code Rate Optimization" block, which will generate the optimized coding rates $r_{0}, \cdots, r_{m-1}$ for the bitplanes $u_{0}, \cdots, u_{m-1}$, respectively. Afterwards, each bitplane $u_{i}(0 \leq i<m)$ is encoded as follows:

1. The bitplane $u_{i}$ will be linearly indexed to generate the sequence $\bar{u}_{i}$ by the block $L$.

2. The resultant sequence $\bar{u}_{i}$ is then encoded by the FEC encoder $i$, which generates the encoded bit sequence $x_{i}$.

Finally, the bit sequences $x_{0}, \cdots, x_{m-1}$ are concatenated into a joint bitstream for transmission.

The receiver structure generates the soft information $y_{0}, \cdots, y_{m-1}$ for the bitplanes $u_{0}, \cdots, u_{m-1}$, respectively. Then each bitplane $u_{i}(0 \leq i<m)$ is estimated as follows:

1. The soft information $y_{i}$ is decoded by the FEC decoder $i$ generating the bit sequence $\hat{\bar{u}}_{i}$, which is the estimated version of bit sequence $\bar{u}_{i}$.

2. The sequence $\hat{\bar{u}}_{i}$ will then be reformatted to the bitplane $\hat{u}_{i}$ by the the block $L^{-1}$, where $\hat{u}_{i}$ is the estimated version of the bitplane $u_{i}$.

Finally, the estimated bitplanes $\hat{u}_{0}, \cdots, \hat{u}_{m-1}$ are reconstructed into the final estimated hologram $\hat{U}$ by the "MUX" block. 

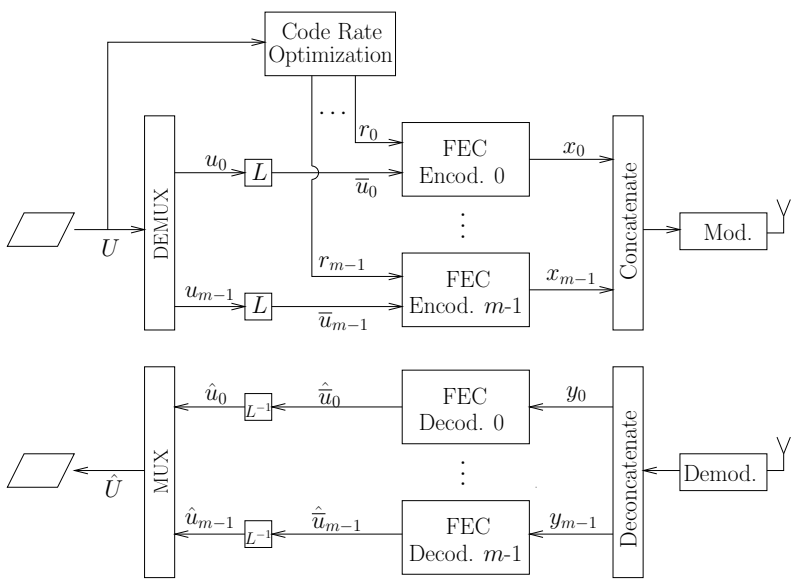

Fig. 1: Block diagram of the proposed Opt-UEP-FEC system.
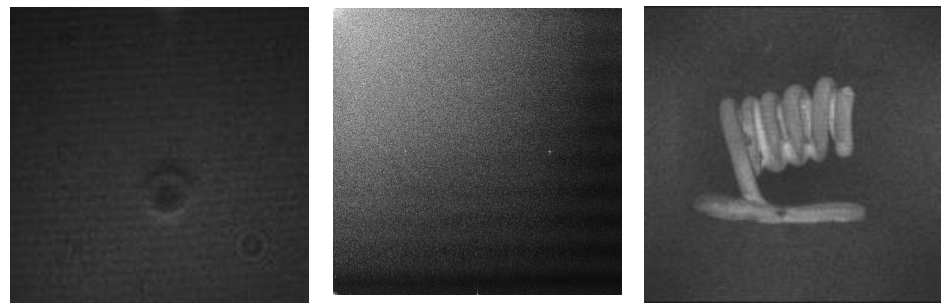

Fig. 2: Example hologram used in the simulations (L-R): hologram of a coil, DCT domain from this Coil hologram, reconstructed image from this Coil hologram

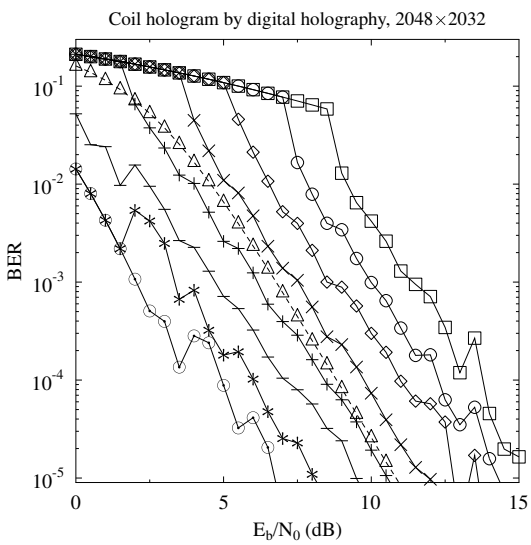

(a) BER vs $E_{b} / N_{0}$ - Coil

$$
\triangle \text { Opt-UEP-RSC }
$$

$-A$ - EEP $\rightarrow$ Opt-UEP-RSC-Est

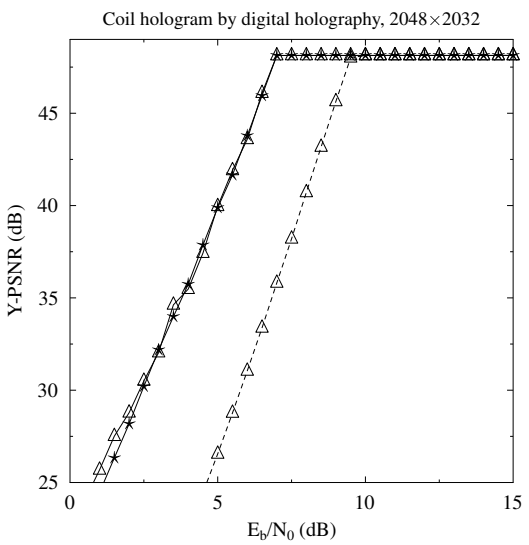

(b) PSNR vs $E_{b} / N_{0}$ - Coil

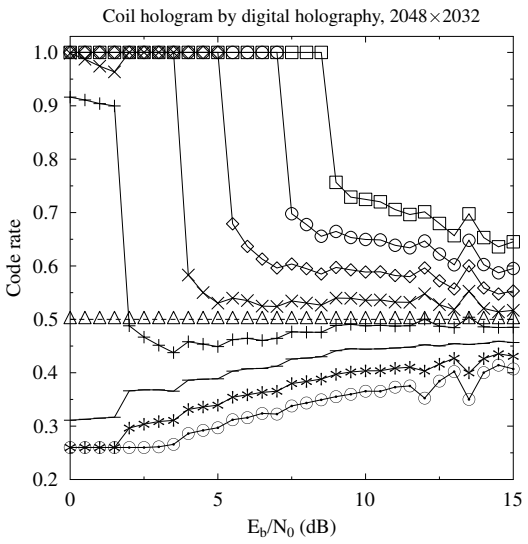

(c) Code rate vs $E_{b} / N_{0}-$ Coil

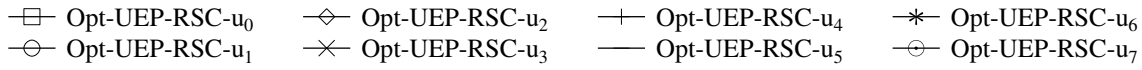

Fig. 3: BER, PSNR, Code rate versus $E_{b} / N_{0}$ performance comparison of the proposed system and the benchmarkers, namely the EEP-RSC scheme, the Opt-UEP-RSC scheme and the Opt-UEP-RSC-Est scheme for the Coil hologram. 
The "Code Rate Optimization" block of Fig. 1 has the task of finding the specific FEC coding rates $r_{0}, \cdots, r_{m-1}$ required for encoding the different-significance bitplanes $u_{0}, \cdots, u_{m-1}$. We denote the position of a specific pixel by $\rho=(w, h)$ in the intensity hologram frame for notational simplicity.

The coding rates $r_{0}, \cdots, r_{m-1}$ of Fig. 1 aim for maximizing the quality of the image reconstructed from the estimated hologram $\hat{U}$ at the receiver. In this paper, our objective is to maximize the peak signal-to-noise ratio (PSNR) of the estimated hologram $\hat{U}$, which represents the most popular objective video quality metric of the reconstructed image [7]. Defining the PSNR of the estimated hologram $\hat{U}$ as $P S N R_{U}$, our objective function (OF) invoked for maximizing the quality of this hologram may be formulated as

$$
\underset{r_{0}, \cdots, r_{m-1}}{\arg } \max \left\{E\left(P S N R_{U}\right)\right\},
$$

where the $P S N R_{U}$ of the reconstructed hologram $\hat{U}$ may be calculated as

$$
P S N R_{U}=10 \cdot \log _{10}\left\{\frac{\left(2^{m}-1\right)^{2}}{M S E}\right\} d B \quad, \quad M S E=\frac{1}{W \cdot H} \sum_{w=0}^{H-1} \sum_{h=0}^{W-1}[U(\rho)-\hat{U}(\rho)]^{2},
$$

where the MSE is calculated based on the original hologram $U$ and the reconstructed hologram $\hat{U}$.

\section{Simulations}

We benchmark our Opt-UEP-RSC system against the traditional EEP-RSC system. The BER versus $E_{b} / N_{0}$ curves of the eight bitplanes of the Coil hologram are displayed in Fig. 3a. As expected, the BER of the bitplanes $u_{4}, \cdots, u_{7}$ of the Opt-UEP-RSC system is always better than that of the EEP-RSC system, while the BER of the bitplanes $u_{0}, \cdots, u_{3}$ is worse than that of the EEP-RSC system owing to the specific code rates.

The PSNR versus $E_{b} / N_{0}$ performance recorded for the Coil hologram is displayed in Fig. $3 \mathrm{~b}$, where the PSNR is also provided by the curve Opt-UEP-RSC-Est. We observe that the Opt-UEP-RSC scheme substantially outperforms the EEP-RSC system, while it has similar performance to the theoretical curve Opt-UEP-RSC-Est. Specifically, the Opt-UEP-RSC scheme achieves an $E_{b} / N_{0}$ reduction of about $2.6 \mathrm{~dB}$ compared to the EEP-RSC scheme at a PSNR of $48 \mathrm{~dB}$. Alternatively, about $12.5 \mathrm{~dB}$ of PSNR hologram quality improvement is observed at an $E_{b} / N_{0}$ of $7 \mathrm{~dB}$. The optimized coding rates found by our proposed regime for the Coil holograms are shown in Fig. $3 \mathrm{c}$.

\section{Conclusions}

We proposed a UEP-FEC technique for the bitplane based transmission of digital holograms over wireless channels, where the coding rates of different bitplanes were optimized to improve hologram quality. We solved the resultant multi-dimensional optimization problem of generating the optimal coding rates for the $m$ bitplanes. Our numerical simulations show that the proposed Opt-UEP-FEC system outperforms the traditional UEP-FEC system by up to 2.6 $\mathrm{dB}$ of $E_{b} / N_{0}$ or $12.5 \mathrm{~dB}$ of PSNR, when employing a RSC code.

Acknowledgements Financial support from an ERC Advanced Fellow Grant, and Science Foundation Ireland (SFI) under Grant Number 13/CDA/222, is gratefully acknowledged.

\section{References}

1. D. Gabor, “A new microscopic principle,” Nature 161, 777-778 (1948).

2. Y. N. Denisyuk, "Photographic reconstruction of the optical properties of an object in its own scattered radiation field," Soviet Physics Doklady 7 543-545 (1962).

3. E. N. Leith and J. Upatnieks, "Wavefront reconstruction with diffused illumination and three-dimensional objects," JOSA B 54, 1295-1301 (1964).

4. J. W. Goodman, "Digital image formation from electronically detected holograms," Appl. Phys. Lett. 11, 77-79 (1967).

5. L. Yaroslavskii and N. Merzlyakov, Methods of Digital Holography. Consultants Bureau, 1980.

6. R. D. Leonardo and S. Bianchi, "Hologram transmission through multi-mode optical fibers," Opt. Express 19, 247-254 (2011).

7. Q. Huynh-Thu and M. Ghanbari, "Scope of validity of PSNR in image/video quality assessment," Electron. Lett. 44, 800-801 (2008). 